\title{
Coordinated Optimal Control of Multiple Reactive Power Devices at Different Voltage Levels in UHVDC Near Zone
}

\author{
Zongzu Yue ${ }^{1, *}$, Xuhui Shen ${ }^{2}$, Feng Yan ${ }^{1}$ \\ ${ }^{1}$ North China Electric Power University, Baoding, China \\ ${ }^{2}$ China Electric Power Research Institute, Beijing, China
}

\begin{abstract}
Affected by different steady-state reactive power output ratios among generators, capacitors and other reactive devices in the end-to-end power grid, voltage collapse may occur due to the failure of the receiving-end AC system, and the problem of voltage stabilization in multi-DC feed systems is particularly common. For suppressing voltage collapse, sufficient dynamic reactive power support is an effective measure, and there are some differences in the dynamic support effect of different reactive power sources. The dynamic reactive power response of the generator and its reactive power margin are two important factors affecting the coordination and optimization of the reactive power of the generator. The comprehensive evaluation index is adopted to optimize the sequencing of the reactive power output of the generator near the DC drop point. A coordinated control method of dynamic and static reactive power for DC near-point systems at different voltage levels is proposed. By controlling the steady-state reactive power output ratio between multiple reactive devices, the node voltage is maintained near the target value, and reactive power control schemes at different voltage levels can be given to meet load changes. Finally, taking the actual situation of Central China Power Grid as an example, the results of different reactive voltage control strategies are compared and analyzed, which proves that the coordinated control strategy of multiple reactive power devices can significantly improve the stability of the receiving grid voltage.
\end{abstract}

\section{Introduction}

The problem of voltage stability at the receiving end is increasingly prominent, which has attracted widespread attention. China's energy distribution is more in the west, less in the east, more in the south, and less in the north, which determines the long-distance, large-capacity DC transmission in China. Although the multi-DC feed-in system solves the power transmission problem of the large load center, in addition, as the proportion of power received outside the receiving network increases year by year, it poses a huge challenge to maintaining the stability of the receiving system. The UHVDC transmission receiving end system needs a large amount of reactive power support, and the required compensation amount reaches $40 \%$ to $60 \%$ of the active power it transmits. Based on the principle of reactive power local compensation, this part of the reactive power demand is provided by the receiving end $\mathrm{AC}$ system ${ }^{[1]}$. The failure of the receiving AC line may cause multiple DCs to fail in phase commutation successively or simultaneously, and even cause the interruption of the DC blocking transmission power, resulting in a large power shortage in the receiving network, which may cause voltage instability or even voltage collapse ${ }^{[2]}$.

Scholars at home and abroad have done a lot of research on the coordinated optimization of reactive devices. The development trend of UHV regional interconnection has also brought severe tests to the system's reactive voltage management capability, especially the dynamic reactive power compensation capability ${ }^{[3]}$; In literature[4], the influence of DC feed on the stability of the receiving end grid voltage was analyzed, and the reactive power optimization analysis of the AC / DC interconnection system of China Southern Power Grid was performed by the plane constraint method. This method, however, takes the squared sum of node voltage deviations as the minimum and does not fully consider the effects of each reactive device on the transient stability; In [5], through the simulation system, the correlation matrix of the transient voltage response of other nodes after the N-1 failure of the target node is constructed, and the correlation matrix is calculated and analyzed using the $\mathrm{K}$-core decomposition method to select nodes with a high degree of correlation with the target node as a transient voltage strong support node; In Reference [6], although the proposed dynamic reactive power configuration scheme can reduce the number of DC commutation failures, no clear index is given on how to distribute the reactive power output; Taking measures from the aspects of control protection, adding additional equipment, and inverter topology reform can effectively improve voltage stability ${ }^{[7]}$. These measures, however, require more capital equipment, and for dynamic reactive power compensation devices, the cost is even higher. It has been found in actual engineering that if the receiving

\footnotetext{
*Zongzu Yue: 740353188@qq.com
} 
end system has a stronger reactive power support capability on the inverter side, the anti-disturbance capability of the DC system will be stronger ${ }^{[8-9]}$; The generator is not only the only active power source in the power system, but also the most important dynamic reactive power source in the system, whose dynamic reactive power reserve is critical to the stability of the transient voltage of the power system. In literature[10-11], it can be seen that by reducing the steady-state reactive power output of the generator and increasing the reactive power reserve of the generator, only this will not only be detrimental to the recovery of the transient voltage after a fault, but also cost a lot. Sufficient dynamic reactive power support is the most effective guarantee for voltage stability at the receiving end. In addition, the different reactive power ratios between multiple reactive devices make the power grid's anti-shock capability very different. Therefore, utilizing existing reactive devices in a reasonable way to improve the stability of the receiving end voltage is an urgent problem.

Based on the dynamic reactive power response of generators and capacitors, in this article, a coordinated optimization control technology for multiple reactive power devices at different voltage levels in the context of multiple DC feeds is proposed. By calculating the dynamic reactive power response and dynamic reactive power support margin of the generator, a comprehensive evaluation index of the voltage stability of each generator near the DC drop point was found to determine the coordinated control sequence of each generator, and through the proposed coordinated optimization control method, a quantitative index is given for the reactive power ratio at each voltage level.

\section{Influence of reactive power source on voltage stability of receiving network}

\subsection{Impacts of reactive power of generator on transient voltage stability of receiving end power grid}

The generator set is equipped with an excitation control system. When the generator terminal voltage drops due to grid failure, the excitation system will quickly adjust the generator excitation voltage according to the magnitude of the voltage deviation, and inject capacitive reactive power into the grid by increasing the internal potential, thereby suppressing the grid voltage drop. The simplified formula of the automatic excitation regulation system is as follows:

$$
-\Delta U_{G} \times \frac{K_{e}}{1+T_{e} p}=\Delta u_{f}
$$

Where Negative sign indicates that the excitation voltage increases when the terminal voltage decreases, $\mathrm{K}_{e}$ and $\mathrm{T}_{e}$ are equivalent magnifications and time constants, $\Delta U_{G}$ is the difference between the generator terminal voltage changes, and $\Delta u_{f}$ is the difference between the excitation voltage changes

In the system of mixed reactive power source of generator set and compensation capacitor, if there are multiple AC channels or AC / DC mixed channels between the system and the main network, then when one of the AC channels or DC channels is broken, the current will be transferred, causing the system voltage to drop. Affected by this, the reactive power output of the generator set and the compensation capacitor will adjust itself and find a new reactive voltage balance. However, at the same voltage level, the voltage drop of the bus voltage after the fault will be equal by the way of generator voltage regulation and capacitor voltage: that is, the value of $\Delta U_{G}$ is equal, and it is further concluded that $\Delta u_{f}$ is equal. As a result, after the voltage regulation method with high initial excitation voltage is disturbed, the excitation voltage will still be high. Without reaching the upper limit of reactive power output, the generator can activate more reactive power at the moment of failure, and it has a stronger ability to support dynamic voltage. When the generator voltage drops sharply due to a short circuit in the system, if the saturation expression is ignored, as in equation 2 , the generator takes strong excitation measures, and the excitation voltage changes from $u_{f}$ to the maximum $u_{f m}$.

$$
\mathrm{u}_{f m}=u_{f}+T_{f} \frac{d u_{f}}{d t}
$$

Under normal operation mode, the more capacitive reactive power output of the generator, the greater the capacitive reactive power output to the grid during the dynamic process. As shown in Figure 1, it can be seen that during normal operation, the larger the output capacitive reactive power of the generator, the higher its internal potential can be maintained (from E1 to E2). When the power grid fails, the voltage at the generator end changes from $\mathrm{U}$ to $\mathrm{U}^{\prime}$, and the voltage differences corresponding to the initial internal potentials E1 and E2 of the generator will be $\Delta \mathrm{E} 1$ and $\Delta \mathrm{E} 2$, respectively. Therefore, the larger the initial internal potential, the greater the difference between the internal potential and the terminal voltage after a fault. From the generator's injection of reactive power into the grid, and its derivative formula with the generator's internal potential, it can be seen that the larger the internal potential, the stronger the generator's ability to inject reactive power into the grid, and the rate of change and regulation Larger, meaning the response will be more responsive.

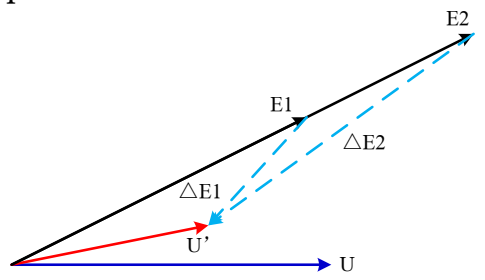

Fig. 1. Influence of the potential of the generator during steady-state operation on the transient voltage difference

$$
\begin{aligned}
& Q=E(E-U \cos \delta) \\
& \frac{d Q}{d E}=\frac{2 E}{X}-\frac{U}{X} \cos \delta
\end{aligned}
$$




\subsection{Effect of different proportions of multiple reactive devices on transient voltage stability of receiving end power grid}

In the following, two different steady-state voltage regulation schemes for capacitive compensation of generators and power grids will be studied, and their effects on the dynamic characteristics of the power grid after disturbance. The simulation test system is established, and the sending end is a generator group composed of multiple units. At the high-voltage side, a capacitive reactive power compensation device for voltage regulation is connected, and the sending-end system is interconnected with the receiving-end infinite system via a double circuit.

The reactive power output of the generator is used, or the capacitance compensation in the power grid is used to control the "controlled bus" to the expected value of 1.02 p.u. In two different control modes, the power flow diagrams of the power grid are shown in Figures 2 and 3, respectively.

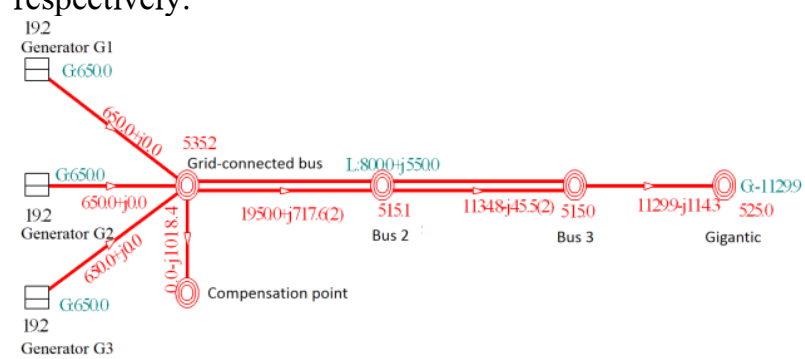

Fig. 2. Scheme 1 Voltage regulation by grid capacitive reactive power compensation

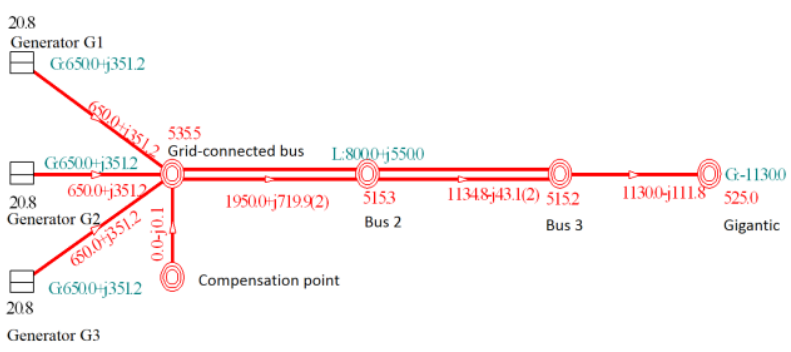

Fig. 3. Scheme 2 Capacitive reactive voltage regulation by generator output

From the figure we can see that under the voltage regulation mode of the capacitive reactive power compensation device, the capacitor reactive power output is 1018.4MVar. In the mode of capacitive reactive power output voltage regulation of the three generating units at the sending end, the output of each unit is 351.2MVar, which totals 1053MVar. Under the two control modes, the voltage of each node of the system and the power flow of the branch are basically the same.

Without considering the regulation effect of the excitation system of the generating unit, if the nonmetallic three-permanent single-trip fault occurs on the controlled busbar side of the one-to-one line between the controlled busbar and the busbar 2, then, under two different control modes, the dynamic reactive voltage operation trajectory of the generator set and capacitor set is shown in Figure 4.

Under two different steady-state voltage control modes, It can be seen from the figure that in the two modes of using the generator for steady-state voltage regulation and grid capacitance compensation for voltage regulation, the dynamic reactive voltage of the reactive source has the following characteristics:

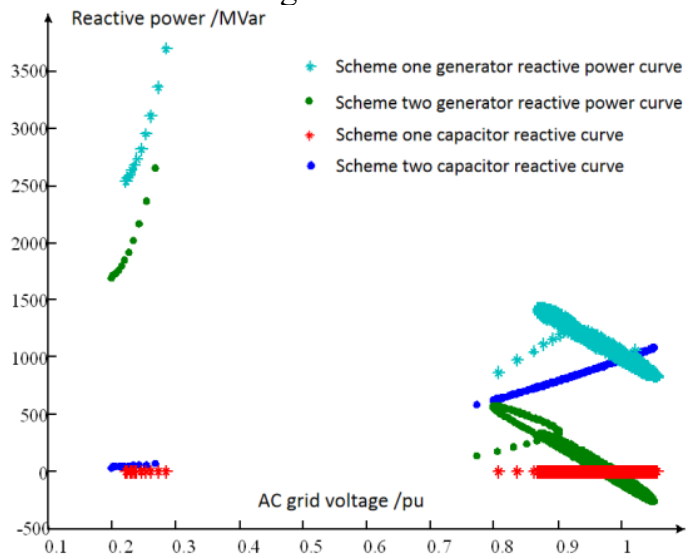

Fig. 4. Reactive source voltage and reactive dynamic trajectory

- In the three stages before the fault, during the fault, and after the fault is cleared, the reactive voltage trajectory of the generator changes as counterclockwise. When a fault occurs, the grid voltage drops sharply. Due to the law of conservation of magnetic flux, the potential in the generator cannot change suddenly at the moment of the fault, so the drop in the grid voltage increases the voltage difference between the potential in the generator and its terminal voltage, and then the capacitive reactive power output by the generator to the grid is dramatically improved in an instant.

- During the fault, the generator can still maintain a large reactive power output. Then after the fault is removed, the grid voltage returns to a high level, and the reactive power output of the generator will basically return to the normal operating level, and the reverse adjustment will be performed with the grid voltage fluctuation, which is beneficial to the voltage recovery and stability.

- Under normal operation mode, the more capacitive reactive power output of the generator, the greater the capacitive reactive power output to the power grid during its dynamic process.

\section{Quantitative indicators}

\subsection{Supporting indicators of generator dynamic reactive power}

Due to the feeding of DC, the reactive power shortage after the large disturbance at the receiving end grid is aggravated. The generator is both the only active power source and the most basic reactive power source. The reactive power supplied by the generator is not infinitely adjustable. In addition, the dynamic reactive power response to the fault varies with the location of the 
generator. If the generator can quickly provide a large amount of reactive power during fault recovery, it will help the grid voltage recovery. As shown in the figure 5, it is a simple power system

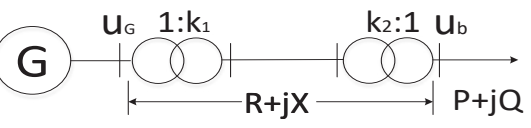

Fig. 5. Simple power system

The generator supplies power to users through step-up transformers, lines and step-down transformers. The voltage at load node $b$ is shown in the formula:

$$
\begin{gathered}
U_{b}=\left(U_{G} k_{1}-\Delta U\right) / k_{2} \\
U_{b} \approx\left(U_{G} k_{1}-\frac{P R+Q X}{U}\right) / k_{2} \cdots \cdots
\end{gathered}
$$

In order to improve $U_{b}$, the voltage regulation of the generator is given priority in various voltage regulation measures, when the terminal voltage of the modern synchronous generator deviates from the rated value by no more than $\pm 5 \%$, it can still run at the rated power. For this type of pressure regulation, no additional equipment is needed, which is the most economical and reasonable pressure regulation measure. For the same compensation point, the electrical distance is reflected in the equivalent reactance $\mathrm{R}+\mathrm{j} X$. The electrical distance is smaller than the reactance value of the unit. Under the same reactive power output, compared with units with longer electrical distances, it has stronger support capabilities. Besides it can also avoid the increase of power loss due to largescale long-range reactive power transmission.

In order to evaluate the dynamic reactive power support capability of the generator, the value of the reactive power incremental standard during the system recovery phase is integrated. The larger the integral value is, the stronger the supporting effect is, and the more the unit that tends to increase the voltage at the machine end is preferred. The integral expression is as follows.

$$
Q_{i}=\int_{t_{0}}^{t_{1}} \frac{Q_{i}(t)-Q_{0 i}}{S_{B}} d t
$$

Where $t_{0}$ refers to the moment of fault removal; $t_{1}$ refers to the cut-off time of generator reactive power integration; $Q_{0 i}$ refers to the initial reactive power of generator i; $Q_{i}(t)$ refers to the generator i Reactive power output; $Q_{i}$ refers to the integral value of the reactive power output of generator $i$ during the integration time.In order to more intuitively observe the transient support effect of different units, the above-mentioned integral value $Q_{i}$ is normalized:

$$
Q_{i}^{\prime}=\frac{Q_{i}}{Q_{\text {imax }}}
$$

In the formula, $Q_{i}^{\prime}$ is the integral value of the reactive power output of the normalized generator node. The larger the integral value, the stronger the support effect.

\subsection{Optimal selection index of generator}

The actual dynamic response of each generator in a certain fault in the actual power grid is different, and the maximum reactive power $Q_{\mathrm{fmax}}$ that can be supported is also different. In order to reasonably optimize the operating state of the generator, not only the dynamic reactive power response of the generator under a certain fault needs to be evaluated, such as the above formula, but also the dynamic reactive power support capacity margin of the generator must be evaluated, as shown in the following formula:

$$
Q_{\text {iymax }}=Q_{i f \max }-Q_{\text {ismax }}
$$

Where: $Q_{\text {iymax }}$ is the margin of dynamic support capacity of generator $\mathrm{i} ; Q_{\text {ifmax }}$ is the maximum dynamic reactive power output of generator i; $Q_{i s m a x}$ is the maximum dynamic reactive power output of generator $i$ after a fault.

To facilitate analysis and keep the index value between $0-1, Q_{\text {iymax }}$ is normalized:

$$
Q_{\text {iymax }}^{\prime}=\frac{Q_{\text {iymax }}}{Q_{\text {ifmax }}}
$$

The transient reactive power response and reactive power margin of the generator should be comprehensively considered. Generator sets with high dynamic reactive power response value and large reactive power margin should be preferentially selected for optimized control. The comprehensive evaluation indicators are shown in the formula:

$$
\mathrm{Z}_{i}=Q_{i}^{\prime} \times Q_{\text {iymax }}^{\prime}
$$

He comprehensive evaluation index $\mathrm{Z}_{i}$ is sorted. The larger the value, the more it indicates that it tends to increase the generator voltage unit.

\section{Coordinated optimization control method of multiple reactive power devices under different voltage levels}

According to the study of the above mechanism, a multireactive power system coordinated optimization control method is proposed for the problem of the multi-feed DC power transmission system arrangement by analyzing the characteristics of different reactive power devices. The optimization method flowchart is shown in the figure 6 :

When the first check result indicates that the DC rupture into the $\mathrm{AC}$ power grid is in a system instability state, the DC receiving end near-site spinning capacity is increased according to a first preset adjustment strategy. The number of start-ups of generators near the DC drop point is increased, the reactive power output of the capacitor is reduced, and the overall operating voltage of the system that the DC breaks into the AC grid is maintained. The current number of starts is the minimum number of starts of the generator.

When the second check result indicates that the DC ruptured $\mathrm{AC}$ power grid is in an unstable state of the system, the current operating voltage of the entire system is determined according to the operation mode when the DC ruptured AC power grid was in a system stable state 
Dynamic and static reactive power coordination control strategy. If the electrical distance between the two DC drop points is short, the same optimized target unit exists, and optimized control is performed according to the higher value of the required terminal voltage.

This optimization method can propose a dynamic and static reactive power coordination control strategy by analyzing the fault of the multi-feed DC transmission system, including: the current minimum system startup number of the generator, the minimum generator terminal voltage of the generator and the reactive power output of the corresponding capacitor under the current overall operating voltage of the system.

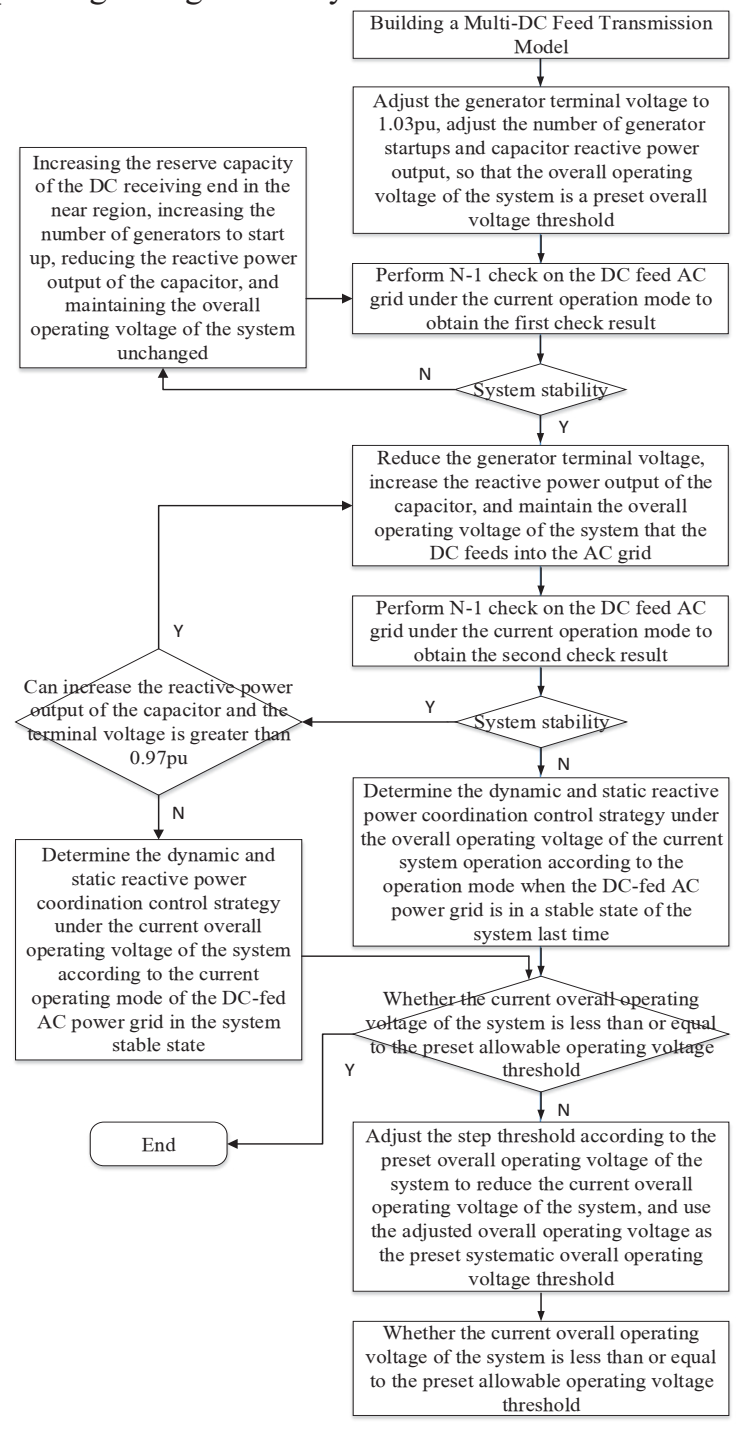

Fig. 6. Optimization method flowchart

\section{Simulation analysis}

\subsection{Simulation analysis of Qishao DC near zone}

Taking the near area of Qishao DC drop point as an example, in the PSD-BPA program, the startup mode of Qishao DC near area is adjusted. By reducing the output of the generator in the near area of Qishao DC and increasing the start-up of the Hubei unit, the $500 \mathrm{kV}$ bus voltage in the near area of Qishao DC is reduced. The comprehensive evaluation indicators of the generator are shown in the table. The voltage of the $500 \mathrm{kV}$ bus in the near area of Qishao DC is controlled to $515 \mathrm{kV}$. The comprehensive evaluation index of the generator in the near area after Qishao DC is closed is shown in the table. The generator terminal voltage in the near zone is divided into two control modes: $0.98 \mathrm{pu}$ and $0.97 \mathrm{pu}$. When the terminal voltage in the near area is $0.98 \mathrm{pu}$, according to the calculated value of the comprehensive evaluation index, the terminal voltage of the Xianglianyuan 01, Xianglianyuan 02, Xiangchangsha 01, and Xiangchangsha 02 units is preferentially reduced to $0.97 \mathrm{pu}$, and the substation capacitor bank input was increased. The following table shows the power-on of the nearby area of Qishao DC and the reactive power compensation of each $500 \mathrm{kV}$ substation.

Table 1 Comprehensive evaluation indicators

\begin{tabular}{|c|c|c|c|c|c|}
\hline Generator & $Q_{i}^{\prime}$ & $Q_{\text {isma: }}$ & $Q_{\text {iymax }}$ & $Q_{\text {iymax }}^{\prime}$ & $\mathrm{Z}_{i}$ \\
\hline Youxian02 & 0.536 & 0 & 372 & 1.000 & 0.536 \\
\hline Changsha01 & 0.517 & 153 & 219 & 0.588 & 0.304 \\
\hline Changsha02 & 0.509 & 146 & 246 & 0.628 & 0.325 \\
\hline XiangtanB3 & 0.544 & 141 & 231 & 0.621 & 0.338 \\
\hline Yiyang03 & 0.541 & -26 & 398 & 1.070 & 0.578 \\
\hline Lianyuan01 & 0.483 & 78 & 108 & 0.581 & 0.281 \\
\hline Lianyuan02 & 0.482 & 74 & 112 & 0.602 & 0.290 \\
\hline
\end{tabular}

Table 2 Qishao DC near-zone generator output (unit MVar)

\begin{tabular}{|c|c|c|c|c|}
\hline \multirow{2}{*}{ Generator } & \multicolumn{2}{|c|}{$0.98 \mathrm{pu}$} & \multicolumn{2}{c|}{$0.97 \mathrm{pu}$} \\
\cline { 2 - 5 } & Output & $\begin{array}{c}\text { Dynamic } \\
\text { reserve }\end{array}$ & Output & $\begin{array}{c}\text { Dynamic } \\
\text { reserve }\end{array}$ \\
\hline Changsha01 & 153 & 219 & 140 & 232 \\
\hline Changsha02 & 146 & 246 & -25 & 397 \\
\hline Lianyuan01 & 78 & 108 & 65 & 121 \\
\hline Lianyuan02 & 74 & 112 & 60 & 126 \\
\hline
\end{tabular}

Table 3 Input of reactive power compensation for $500 \mathrm{kV}$ substations in Qishao DC near-zone (unit: MVar)

\begin{tabular}{|c|c|c|c|}
\hline Substation & $\begin{array}{c}\text { Reactiv } \\
\text { e power } \\
\text { compen } \\
\text { sation }\end{array}$ & $\begin{array}{c}\text { Reactive power } \\
\text { configuration } \\
\text { (machine } \\
\text { terminal } \\
\text { voltage 0.98pu) }\end{array}$ & $\begin{array}{c}\text { Reactive } \\
\text { power } \\
\text { configuration } \\
\text { (machine } \\
\text { terminal } \\
\text { voltage } \\
0.97 \mathrm{pu})\end{array}$ \\
\hline Heling & $4 \times 60$ & -- & -- \\
\hline Guting & $2 \times 60$ & -- & -- \\
\hline Yuntian & $9 \times 40$ & -- & $2 \times 40$ \\
\hline Chuangu & $4 \times 60$ & -- & $2 \times 40$ \\
\hline Xingcheng & $7 \times 60$ & -- & -- \\
\hline Aijia & $6 \times 60$ & -- & $2 \times 60$ \\
\hline Liuyang & $5 \times 60$ & -- & -- \\
\hline Hengyang & $2 \times 60$ & -- & -- \\
\hline
\end{tabular}

For $500 \mathrm{kV}$ line Shaoshan to Heling line N-1 failure, two voltage regulation control methods are adopted to have different grid voltage stability. The voltage fluctuation of $500 \mathrm{kV}$ bus in Shaoshan and the comparison of reactive power output of Youxian 02 are shown in the figure 7 and figure 8 . 


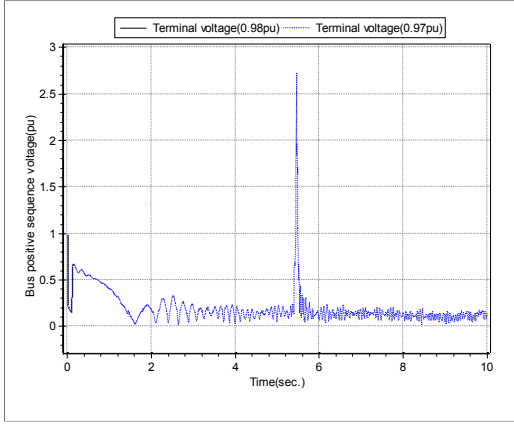

Fig 7 Voltage fluctuation curve of Shaoshan $500 \mathrm{kV}$ bus after N-1 failure at Shaoshan to Heling line

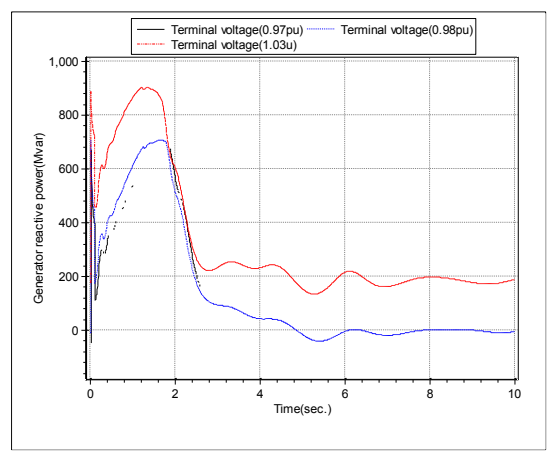

Fig 8 Reactive power of Youxian 02 after N-1 failure on the Shaoshan-Heling line

The same voltage level is maintained in the near area of Qishao DC. In the case of Youxian 02 with different steady-state reactive power output, after the N-1 failure of the Shaoshan-Heling line, with the increase of the generator terminal voltage, the difference between the peak value of the reactive output fluctuation and the steady state value after the fault of Youxian 02 in the three cases is 707MVar, 708MVar, 704MVar. The simulation results show that at the same voltage level, the larger the steady-state reactive power output of the generator, the stronger the ability of dynamic reactive power output during the transient process after a fault; That is, at the same voltage level, the higher the proportion of voltage regulation by the generator, the higher the voltage stability level of the entire system, and the worse it is the opposite. The simulation results of the actual system are consistent with the results of the aforementioned mechanism analysis.

\section{Conclusion}

In this paper, in the context of multiple DC feeds, aiming at the coordinated optimization control of the existing reactive power devices in the power grid, a dynamic and static reactive power coordinated control method for multiple DC feed systems at different voltage levels was proposed to improve the system's anti-shock capability. Based on the mechanism analysis of the generator excitation control system, it is pointed out that the larger the generator 's output capacitive reactive power during normal operation, the higher its internal potential. When the AC system fails, the higher the excitation voltage of the generator, the dynamic reactive power output increases, and the response is faster. After comprehensive consideration of generator transient reactive power support effect and reactive power margin, a comprehensive evaluation index is formed. Through different ratios with the parallel capacitors, the node voltage is controlled to different voltage levels, and the quantitative index of the reactive power ratio between multiple reactive devices at each voltage level is given. The simulation and comparison verification of Central China Power Grid proves that the proposed method can effectively improve the stability of the receiving end grid voltage and reduce the rotating reserve capacity. This method can be applied to the formulation of offline schemes and the online monitoring of voltage stability.

\section{References}

1. X. Y. Li, H. B. Liu, "The Influence of the Proportion of the Capacity of the Transmitter System on the Failure of DC Commutation and Its Countermeasures," Proceedings of the CSEE, 39 (2019)

2. W. Qiu, J. B. He, "Transient voltage stability analysis of Hunan Power Grid with infeed UHVDC," Electric Power Automation Equipment, 39 (2019)

3. H. Xiong, T. Y. Xiang, X. Zhan. "An Optimal Control Strategy of Reactive Power and Voltage for UHVAC Power Transmission System," Power System Technology, (2012)

4. X. Li, Z. B. Yang, Y. Yuan. P. T Xu,S. J. Lin,Z. J. Liang,C. X. Chen. "Multi-objective reactive power optimal control of AC-DC systems including power loss characteristics of converter stations," Electric Power System Protection and Control, 45 (2017)

5. H. H Qiong, D. Ma, Y. T. Wang,X. J. Guo,Y. C. Zhang, "Site Selection Method for Transient Voltage Control for Receiving End of HVDC System Based on Complex System Theory," Power System Technology, 40, 10 (2016)

6. H. L. Zhang, F. S. Liu, W. Li, "Site selection for dynamic reactive power compensaction and improvement of recovery from commutation failures in multi-infeed HVDC system," Automation of Electric Power Systems, 40, 5 (2016)

7. Y. Yuan, Z. N. Wei, X.Lei, "Survey of commutation failures in DC transmission systems," Electric Power Automation Equipment, 33, 11 (2013)

8. S. D. Gao, "The voltage stability characteristic of terminal power grid fed by HVDC," North China Electric Power University, (2018)

9. Y. Zhao, C. Hong, Y. G. Zeng, et al, "The voltage support strength factor (VSF) for multi-infeed HVDC systems and its application in CSG's statcom allocation scheming," Southern power system sechnology, 8, 4 (2014)

10. M. J. Laufenberg, M. A. Pai, "A new approach to dynamic security assessment using trajectory sensitivities," IEEE Transactions on Power Systems, 13, 3 (1998) 
11. M. Zima, G. Anderson, "Stability assessment and emergency control method using trajectory sensitivities," IEEE Bologna Power Tech Conference

Proceedings, (2003) 